\title{
Anesthetic management of a patient with Lennox-Gastaut syndrome with intractable epilepsy
}

\section{-a case report-}

\author{
Mi-Na Park and Ji-Yeon Kim \\ Department of Anesthesiology and Pain Medicine, Samsung Medical Center, Sungkyunkwan University School of Medicine, Seoul, Korea
}

A 34-month-old female patient who had a prior history of generalized tonic-clonic seizures had been diagnosed with the Lennox-Gastuat syndrome. She was scheduled for epiblepharon repairs in both lower lids under general anesthesia. Preoperatively, her tonic seizure was poorly controlled with antiepileptic drugs. General anesthesia with thiopental and isoflurane was provided and the intraoperative course and the emergence of anesthesia were uneventful and smooth. (Korean J Anesthesiol 2013; 65: 353-356)

Key Words: Anesthesia, Epileptic encephalopathy, Lennox-Gastaut syndrome.

Lennox-Gastaut syndrome is a rare epileptic encephalopathy that usually occurs before age 8 , with a peak between 3 and 5 years of age. The reported prevalence rates for Lennox-Gastaut syndrome vary widely between $1-10 \%$ of all childhood epilepsies. Lennox-Gastaut syndrome consists of a triad of cognitive impairments, multiple seizures and an electroencephalography (EEG) that shows slow $(\leq 2.5 \mathrm{~Hz})$ spike-and-wave bursts with abnormal background activity [1,2]. Most patients have tonic seizures during sleep that may be subtle and nearly all have treatment-resistant, lifelong epilepsy. Intractable epilepsy can create difficulties for anesthesiologists, both during anesthetic induction and recovery. Anesthesiologists have to keep in mind that the patients can seizure at any time. Uncontrolled seizures can produce a variety of relevant traumatic injuries to different body areas, such as the head, airway and others. Also, if vomit- ing occurs, airway and gas exchange can become compromised. Hypertension and hypoglycemia can arise as either a cause or result of epilepsy.

This study describes the application of general anesthesia to a patient with Lennox-Gastaut syndrome with intractable epilepsy who had optical surgery. We report successful anesthetic management of a patient with the Lennox-Gastaut syndrome and an accompanying literature review.

\section{Case Report}

The patient was a 34-month-old girl with a height of $90 \mathrm{~cm}$ and weight of $12.5 \mathrm{~kg}$ who was diagnosed with Lennox-Gastaut syndrome who was scheduled for surgical treatment of a lower lid epiblepharon. She was born by normal spontaneous vaginal

Received: December 28, 2011. Revised: 1st, February 3, 2012; 2nd, May 10, 2012; 3rd, October 17, 2012. Accepted: October $25,2012$.

Corresponding author: Ji-Yeon Kim, M.D., Department of Anesthesiology and Pain Medicine, Samsung Medical Center, Sungkyunkwan University School of Medicine, 50, Irwon-dong, Gangnam-gu, Seoul 135-710, Korea. Tel: 82-2-3410-2470, Fax: 82-2-3410-0361, E-mail: jydr. kim@samsung.com

(C) This is an open-access article distributed under the terms of the Creative Commons Attribution Non-Commercial License (http:// creativecommons.org/licenses/by-nc/3.0/), which permits unrestricted non-commercial use, distribution, and reproduction in any medium, provided the original work is properly cited. 
delivery with a weight $3.52 \mathrm{~kg}$ at 38 weeks 6 days and there were no specific abnormalities in the antenatal exam. At the age of 40 days, she began to show signs of generalized tonic-clonic seizures. At that time, there were no abnormalities in her EEG and magnetic resonance imaging (MRI), but video-electroencephalographic monitoring showed lateralized to midline central, regionalized to central. Repeated EEG and MRI were normal and an epilepsy monitoring unit showed hypermotor seizures in the left frontotemporal and central regions.

She displayed developmental delay when compared to toddlers in the same age group. Because of the ataxia and muscle atrophy, she had been bedridden. Although she took antiepileptic drugs (AEDs), the tonic seizures persisted. As an additional treatment, she had given a ketogenic diet for 2 months. However, it also had no effect and was stopped. The frequency of the seizure was about three to ten times per day. Her preoperative vital signs, chest X-ray and laboratory data were normal (Table 1).

The patient took phenobarbital $(30 \mathrm{mg})$, vigabatrin $(300 \mathrm{mg})$, carbamazepine (250-200-100 mg), levetiracetam (250 mg), leucovorin (5 mg), pyridoxine (12.5 mg), lamotrigine (chewable, $12.5 \mathrm{mg}$ ) until the morning of the day of operation. Midazolam $(1.2 \mathrm{mg}, 0.1 \mathrm{mg} / \mathrm{kg}$ ) was administered intravenously for sedation upon entering the operating room. Before anesthesia, the blood pressure was $94 / 60 \mathrm{mmHg}$, heart rate was 89 beats/min and oxygen saturation was $100 \%$. Anesthesia was induced with thiopental sodium ( $50 \mathrm{mg}, 5 \mathrm{mg} / \mathrm{kg}$ ) intravenously and mask ventilation was started with $5 \mathrm{vol} \%$ isoflurane and $100 \%$ oxygen. After checking that the mask ventilation was operating properly, rocuronium bromide $(10 \mathrm{mg}, 1 \mathrm{mg} / \mathrm{kg}$ ) was administered. After a minute, proper muscle relaxation was verified and the glottis was exposed using a Machintosh laryngoscope number 2. The trachea was intubated with an endobronchial tube with a 4.5 $\mathrm{mm}$ envelope. Mechanical ventilation was started with pressurecontrolled ventilation at a pressure $14 \mathrm{cmH}_{2} \mathrm{O}$ (a tidal volume 90-100 $\mathrm{ml}$ ) and a respiratory rate of $18-20$ beats/min. Anesthesia was maintained with isoflurane (1.0-1.2 vol \%) in oxygen (40\%). Monitors included electrocardiography, pulse oxymetry and capnography, and inspired and the end-tidal concentrations of isoflurane were measured. During the operation, the blood pressure was $85-100 / 40-50 \mathrm{mmHg}$, heart rate was $85-110$ beats/

Table 1. Preoperative Laboratory Test

\begin{tabular}{llll}
\hline VBGA & & $\mathrm{Na}(\mathrm{mmol} / \mathrm{l})$ & 137.0 \\
$\mathrm{pH}$, venous $(\mathrm{pH})$ & 7.402 & $\mathrm{~K}(\mathrm{mmol} / \mathrm{l})$ & 4.3 \\
$\mathrm{pCO}_{2}$, venous $(\mathrm{mmHg})$ & 32.4 & $\mathrm{Cl}(\mathrm{mmol} / \mathrm{l})$ & 103.0 \\
$\mathrm{pO}_{2}$, venous $(\mathrm{mmHg})$ & 66.2 & Albumin $(\mathrm{g} / \mathrm{dl})$ & 4.5 \\
$\mathrm{HCO}_{3}$, venous $(\mathrm{mmol} / \mathrm{l})$ & 9.7 & Fasting glucose $(\mathrm{mg} / \mathrm{dl})$ & 86.0 \\
Base excess, venous $(\mathrm{mmol} / \mathrm{l})$ & -4.1 & $\mathrm{BUN}(\mathrm{mg} / \mathrm{dl})$ & 12.2 \\
$\mathrm{O}_{2}$ Saturation, venous $(\%)$ & 93.0 & Creatinine $(\mathrm{mg} / \mathrm{dl})$ & 0.25 \\
\hline
\end{tabular}

VBGA: venous blood gas analysis, BUN: blood urea nitrogen. min, oxygen saturation was $100 \%$ and body temperature was $36.1-36.7^{\circ} \mathrm{C}$. The blood sugar level was $149 \mathrm{mg} / \mathrm{dl}$ during the middle of the operation. The operation lasted a total of about 2 hours and $150 \mathrm{ml}$ Ringer's lactate solution was supplied. At the end of surgery, anesthetics were discontinued. She recovered spontaneous respiration and glycopyrrolate $(0.1 \mathrm{mg}, 0.01 \mathrm{mg} /$ $\mathrm{kg}$ ) and pyridostigmine $(2.5 \mathrm{mg}, 0.2 \mathrm{mg} / \mathrm{kg}$ ) were intravenously injected. After extubation was performed, her respiratory and circulatory conditions were stable and she was transferred to the postanesthetic care unit (PACU). Forty minutes later, during her stay with her mother in the PACU, she had a tonic seizure attack with mild eyeball deviation. The duration of the seizure was thirty seconds and there was no saturation change during the convulsion. According to her mother, the seizure was similar to her usual symptoms. She was discharged from the hospital the following day without complications.

\section{Discussion}

Lennox-Gastaut syndrome is a severe form of epilepsy that usually starts in early to mild childhood. It is characterized by multiple seizure types, abnormal EEG with sole spike-and-wave discharges and cognitive problems. The etiology of the LennoxGastaut syndrome may be either cryptogenic or symptomatic. Approximately $75 \%$ of the cases are thought to be symptomatic, implying an identifiable cause such as a cerebral malformation or hypoxic-ischemic injury. The cryptogenic group (no apparent cause and no neurologic precedents) may account for approximately $25 \%$ of the cases. The Lennox-Gastaut syndrome is a difficult-to-treat epilepsy syndrome $[1,3]$. Treatments of seizures for patients with the Lennox-Gastaut syndrome vary according to the predominant seizure type. In resistant cases, rufinamide, zonisamide, felbamate, levetiracetam, acetazolamide, methsuximide, corticosteroids, a ketogenic diet or intravenous immunoglobulin (IVIG) can be used [3]. In this case, our patient had been treated with phenobarbital, vigabatrin, carbamazepine and levetiracetam, but these drugs had no effects on her tonic seizures and the ketogenic diet was also ineffective.

There are several specific considerations that an anesthesiologist must consider when managing a patient with epilepsy. First, one has to consider carefully the ability of the anesthetics to modulate or potentiate seizure activity. Second, the effects of AEDs and interaction with anesthesia should be considered. Third, one must evaluate the presence of concomitant medical problems occasionally associated with epilepsy [4,5].

Some anesthetics can produce seizures whereas others tend to stop seizure. With different doses or under different physiologic conditions, some anesthetics can do both and function as either a proconvulsant or anticonvulsant.

Most sedative hypnotics affect brain EEG. Propofol is a very 
popular sedative for off-site procedures and has more predictable effects on epileptiform activity. Most reports suggest that propofol produces anticonvulsant effects [6,7]. It has been used to treat refractory status epilepticus and was shown to have a dose-dependent effect on EEG. Barbiturates generally inhibit epileptiform EEG activity and are used to induce burst suppression and electrical silence for status epilepticus. Propofol and thiopental sodium can be used to treat Lennox-Gastaut syndrome. When using propofol, the duration of high doses should be limited to 48 hours and the risk of propofol infusion syndrome should be kept in mind. High doses of barbiturates can effectively terminate seizures but sometimes recovery from anesthesia prolongs ventilator treatment and intensive care. We used thiopental sodium because our patient was young child and admitted postoperatively. Benzodiazepines are potent anticonvulsants commonly used for the treatment of acute seizures. They have consistently been shown to affect EEG activity. However, midazolam, a short acting benzodiazepine, has been reported to be used without problems. We administered midazolam to the patient for anxiolysis and sedation because preoperative anxiety could provoke seizures. For the most part, the various benzodiazepines, although used in the treatment of LennoxGastaut syndrome, are associated with sedation and drooling, and some have been shown to induce tonic seizures in patients with Lennox-Gastaut syndrome [2,3]. Fortunately, our patient did not have these side effects; however, it can occur at any time. Therefore, one must always consider the effects of using benzodiazepines for the treatment of Lennox-Gastaut syndrome. Ketamine causes a variety of effects on EEG and also seems to possess anticonvulsant properties. It has been used to terminate tonic-clonic convulsions as well as febrile convulsions that are unresponsive to conventional antiepileptic therapy $[4,8]$. Opioids generally have less effect on EEG activity. Remifentanil produces dose-related limbic system activation and causes electroconvulsive therapy-induced seizure in humans [4,5,9]. Inhalation anesthetics have diverse effects on epilepsy. There is controversy over the epileptogenic potential of nitrous oxide. When it is used alone, nitrous oxide produces frontally dominant high frequency activity, but when used with halogenated inhalational agents, it can be additive or antagonistic [5]. Other inhalation anesthetics produce dose-related reduction in EEG amplitude and frequency after initial activation, but the degree of depression varies between agents. Isoflurane and desflurane are potent anticonvulsants that are capable of burst suppression at clinically usable concentrations. Burns et al. used isoflurane as an alternative treatment for refractory status epilepticus [10]. Reports of sevoflurane-induced epileptiform activity and abnormal movements have warranted concerns about its use, however, despite these EEG findings, there is a lack of clinical seizure activity. The incidence of abnormal movements during induction or recovery with sevoflurane has been reported to be about 5\% [11]. Deep sevoflurane anesthesia and/or hyperventilation are risk factors. According to these reports, isoflurane and desflurane are safer maintenance inhalation anesthetics than sevoflurane. We used isoflurane because it was considered more appropriate for patient with intractable epilepsy.

Anesthesiologists need to be aware of all common adverse effects of AEDs, including drowsiness, sedation, somnolence and lethargy. The newer AEDs, so called second generation of AEDs, including felbamate, vigabatrin, lamotrigine, gabapentin, topiramate, tiagabine, oxcarbazepine, levetiracetam, pregabalin and zonisamide, offer decreased drug interactions and greater safety [4,5].

There is a lack of information about the effects of prior AED use on anesthesia. It was suggested that drugs that affect hepatic enzymes may alter metabolism of the anesthetic drugs. Predominantly, the first generation AEDs, such as phenytoin, carbamazepine and phenobarbital are resistance to the effects of neuromuscular-blocking agents. These drugs enhance cytochrome P450s, which affects the metabolism of other drugs and may necessitate adjustments to dosing of anesthetics such as propofol, muscle relaxants and opioids. Therefore, epileptic patients receiving AEDs require higher doses of fentanyl to maintain a comparable depth of anesthesia $[5,12]$.

Numerous approaches are currently used to treat the LennoxGastaut syndrome, including the use of AEDs, other drug interventions (corticosteroids and IVIG) and nonpharmacologic treatments (ketogenic diets and vagus nerve stimulation). In particular, anesthetic management must be carefully considered if the patient in on a ketogenic diet, which consists of a high fat and low carbohydrate and protein diet. Sedative syrups containing amino acids and sugar for premedication should be avoided because the infusion of amino acids and glucose has been shown to be associated with a decrease in plasma ketones. Perioperative intravenous fluids must also be amino acid and carbohydrate free. Acid-base imbalances should be carefully monitored and treated immediately, because ketone bodies have the potential to lead to metabolic acidosis $[13,14]$.

In conclusion, Lennox-Gastaut syndrome is characterized by frequent intractable seizures that cannot be treated with AEDs. It is rare that patients with uncontrolled seizure, like our patient, undergo surgeries that do not require general anesthesia. Thus, it is difficult for anesthesiologists to determine the best anesthesia approach for patients with Lennox-Gastaut syndrome with intractable epilepsy. Moreover, there is no established anesthetic management plan. Based on a review of the literature, we recommend using thiopental and isoflurane for anesthetic management. In addition, anesthesiologists should consider preoperative AEDs use and a combination of drugs for patients with Lennox-Gastaut syndrome. 


\section{References}

1. Camfield PR. Definition and natural history of Lennox-Gastaut syndrome. Epilepsia 2011; 52 Suppl 5: 3-9.

2. Crumrine PK. Management of seizures in Lennox-Gastaut syndrome. Paediatr Drugs 2011; 13: 107-18.

3. Ferrie CD, Patel A. Treatment of Lennox-Gastaut Syndrome (LGS). Eur J Paediatr Neurol 2009; 13: 493-504.

4. Kofke WA. Anesthetic management of the patient with epilepsy or prior seizures. Curr Opin Anaesthesiol 2010; $23: 391-9$.

5. Ren WH. Anesthetic management of epileptic pediatric patients. Int Anesthesiol Clin 2009; 47: 101-16.

6. Parviainen I, Kälviäinen R, Ruokonen E. Propofol and barbiturates for the anesthesia of refractory convulsive status epilepticus: pros and cons. Neurol Res 2007; 29: 667-71.

7. Rossetti AO, Reichhart MD, Schaller MD, Despland PA, Bogousslavsky J. Propofol treatment of refractory status epilepticus: a study of 31 episodes. Epilepsia 2004; 45: 757-63.

8. Borris DJ, Bertram EH, Kapur J. Ketamine controls prolonged status epilepticus. Epilepsy Res 2000; 42: 117-22.

9. Kofke WA, Blissitt PA, Rao H, Wang J, Addya K, Detre J. Remifentanil-induced cerebral blood flow effects in normal humans: dose and ApoE genotype. Anesth Analg 2007; 105: 167-75.

10. Fugate JE, Burns JD, Wijdicks EF, Warner DO, Jankowski CJ, Rabinstein AA. Prolonged high-dose isoflurane for refractory status epilepticus: is it safe? Anesth Analg 2010; 111: 1520-4.

11. Voss LJ, Sleigh JW, Barnard JP, Kirsch HE. The howling cortex: seizures and general anesthetic drugs. Anesth Analg 2008; 107: 1689-703.

12. Tempelhoff R, Modica PA, Spitznagel EL Jr. Anticonvulsant therapy increases fentanyl requirements during anaesthesia for craniotomy. Can J Anaesth 1990; 37: 327-32.

13. Ichikawa J, Nishiyama K, Ozaki K, Ikeda M, Takii Y, Ozaki M. Anesthetic management of a pediatric patient on a ketogenic diet. J Anesth 2006; 20: 135-7.

14. Valencia I, Pfeifer H, Thiele EA. General anesthesia and the ketogenic diet: clinical experience in nine patients. Epilepsia 2002; 43: 525-9. 